\title{
Video-EEG Analysis Following Microradiosurgical Transections of Rat Sensorimotor Cortex Seizure Focus
}

\author{
David Anschel ${ }^{1}$, Alberto Bravin ${ }^{2}$, Elke Bräuer-Krisch ${ }^{2}$, Geraldine Le Duc, Pantaleo Romanelli \\ 4 \\ 1. Neurology Department, St. Charles Hospital 2. European Synchrotron Radiation Facility, Grenoble, \\ France 3. 4. Cyberknife Center, Cdi, Milan; European Synchrotron Radiation Facility (esrf), Grenoble, \\ France, AB Medica, Milan;cyberknife Center, Cdi, Milan; European Synchrotron Radiation Facility (esrf), \\ Grenoble, France
}

$\square$ Corresponding author: David Anschel, danschel@yahoo.com

Disclosures can be found in Additional Information at the end of the article

\section{Abstract}

Introduction: Multiple subpial transections are an alternative to resection of the epileptic focus for select patients. Microradiosurgery is a developing technique, which uses submillimetric beams of radiation to treat disease. This preliminary study was designed to evaluate electroencephalography and behavioral findings following microradiosurgical transcortical transection (MTT) of a rat seizure focus.

Materials and Methods: Kainic acid was used to induce a seizure focus within the left sensorimotor cortex of two rats. Before and up to 10 hours following synchrotron-generated MTT, video-EEG was recorded.

Results: Convulsive seizures were eliminated less than five hours after the MTT. Although more subtle electrographic ictal events remained detectable by EEG recording, the propagation of seizures was effectively disrupted.

Conclusions: These results demonstrate the feasibility of recording VEEG before and after precise microbeam irradiation from a synchrotron source. VEEG allowed evaluation of electrographic ictal events, in addition to clinical seizures. The results show some promise that MTT can limit the spread of seizures without causing clinically evident neurological damage.

Received 09/26/2013

Review began 09/29/2013

Published 11/05/2013

๑) Copyright 2013

Anschel et al. This is an open access article distributed under the terms of the Creative Commons Attribution License CC-BY 3.0., which permits unrestricted use, distribution, and reproduction in any medium, provided the original author and source are credited.
Categories: Medical Physics, Neurology, Neurosurgery

Keywords: epilepsy, microbeams, radiation therapy, radiosurgery, synchrotron

\section{Introduction}

Multiple subpial transections (MST) were pioneered in the 1970-1980's to address the need for a surgical approach to epileptic foci in eloquent cortex [1]. The technique involves the use a specialized instrument and methodology to sever horizontal intracortical fibers involved in the propagation of seizures, while preserving vertical fibers and penetrating blood vessels which are essential for brain function. Overall, the method has been useful for the treatment of epilepsy, especially when combined with a partial resection [2]. Although less damaging to the brain than a traditional resection, MST still exposes patients to the risks generally associated with a craniotomy. 
Microradiosurgery is the use of sub-millimetric beams (microbeams) of radiation to treat disease [3-4]. Synchrotron-generated spatially fractionated microbeams have been studied in several preclinical experiments and have the extraordinary property of a rapid dose fall-off. The dose gradient is so rapid that there will be negligible radiation exposure to tissue $0.1 \mathrm{~mm}$ distant from the target. Therefore, arrays of tightly spaced microbeams can be dosed at 100'sto-1000's of Grays (Gy), producing a lethal effect only to those cells within or in the close vicinities of the beam path. Since the X-rays issued from synchrotron radiation sources used for microradiosurgery are of low energy, the tissue half-value layer is small ( $43 \mathrm{~mm}$ with $120 \mathrm{keV}$ microbeams [5], $42 \mathrm{~mm}$ for the ESRF spectrum [6]). This makes the technique ideal for treating superficial brain lesions, as the incidental radiation delivered to non-targeted deep tissues will be substantially less than that absorbed by the target.

Arrays of parallel microbeams therefore may be well-suited for non-invasive microradiosurgical transcortical transections (MTT). This concept was recently explored in a series of preclinical experiments at the European Synchrotron Radiation Facility (ESRF), Grenoble, France [7]. The present report details the methodology and results of recording video-EEG (VEEG) before and after MTT.

\section{Materials And Methods}

The study was approved by the European Synchrotron Radiation Facility, Grenoble, France. issued protocol number protocol number MD-574. All operative procedures related to animal care strictly conformed to the guidelines of the French Government. Male Wistar rats (250-275 g) were purchased from Charles River Laboratories (L'Asbresle, France). Rats were kept under environmentally controlled conditions (ambient temperature $=22^{\circ} \mathrm{C}$, humidity $=40 \%$ ) on a 12 hour light/dark cycle with food and water ad libitum.

Following the induction of general anesthesia with isoflurane (2\%), two male Wistar rats (250$275 \mathrm{~g}$ ) (Charles River Laboratories, L'Asbresle France) were placed in a stereotactic head frame, and the cranium was exposed. Four stainless steel bone screws with attached wires (Plastics One, Roanoke, VA) were implanted over the right and left frontal lobes, and posterior right and left parietal lobes. Reference and ground screws were placed more posteriorly. The wires were connected to a connecting plug, and the plug was affixed to the skull with dental cement. A guide cannula (Bilaney $\mathrm{GmbH}$, Schirmerstr, Germany) was implanted in the left cerebral cortex $(1.0 \mathrm{~mm}$ anterior to the bregma, $2.0 \mathrm{~mm}$ lateral to the midline, and $1.0 \mathrm{~mm}$ ventral from the outer surface of dura mater). Kainic acid $(20 \mu \mathrm{g} / 1 \mu \mathrm{l})$ was slowly injected by a microcannula into the left sensorimotor cortex. The cannula was left in place for two minutes before being removed, at which point general anesthesia was discontinued. VEEG data was recorded immediately following kainic acid injection using Grass Technologies Aura system (Grass Technologies, West Warwick Rhode Island) and a wire-commutator system (Plastics One, Roanoke, VA) allowing the rats to have relatively free movement.

Between two to four hours after injection, VEEG recording was suspended while the rats underwent irradiation. Irradiations were performed at the ID17 biomedical beamline of the ESRF. Details of the general technique have been reported previously [4, 7]. Briefly, X-rays are generated by the magnetic "wiggling" of the path of electrons traveling at near light speed within the synchrotron. At ID17, the so-created X-ray spectrum has a mean energy of $107 \mathrm{keV}$ and range extending, after filtration using $\mathrm{Be}(0.5 \mathrm{~mm}), \mathrm{C}(1.5 \mathrm{~mm}), \mathrm{Al}(1.5 \mathrm{~mm})$, and $\mathrm{Cu}(1.0$ $\mathrm{mm}$ ), from 50 to approximately $350 \mathrm{keV}[6,8]$. The synchrotron X-ray beam is quasi-laminar and is then collimated into an array of rectangular microbeams by a multi-slit collimator placed at $42 \mathrm{~m}$ from the photon source and $1 \mathrm{~m}$ upstream from the head of the experimental animals. The X-ray dose rate was approximately $16,000 \mathrm{~Gy} / \mathrm{s}$; it is directly proportional to the instantaneous electron ring current circulating in the storage ring. 


\section{Cureus}

Rats were fixed by ear bars and teeth on a custom-built Plexiglas stereotaxic frame and placed on a Kappa-type goniometer (Huber, Germany), by which the rat could be translated and rotated in front of the fixed horizontal X-ray beam. The beam height was defined by a $(520 \pm 5)$ $\mathrm{mm}$ tungsten slit, placed at $1 \mathrm{~m}$ upstream of the animal. An array of parallel microbeams was delivered to the area of the left sensorimotor cortex. The targeting setup is shown in Figure 1.
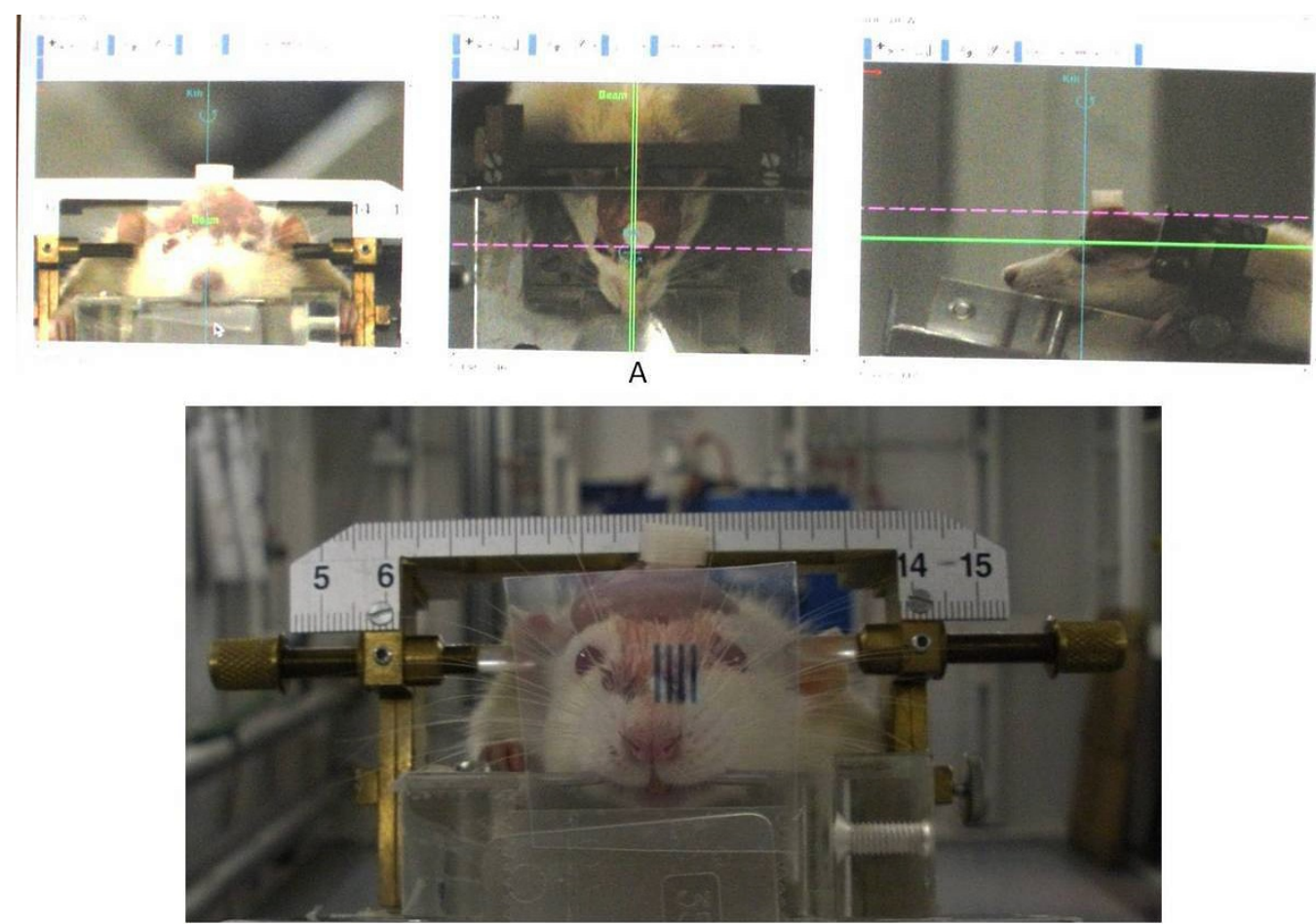

B

\section{FIGURE 1: Video camera shots of the animal fixed by the head frame}

Figure 1A shows video camera shots (from left to right: front, top, lateral) of the animal fixed by the head frame on the kappa goniometer (not visible), capable of micrometric precise movements in three dimensions. The green line represents the microbeam propagation. Figure $1 \mathrm{~B}$ is a post-irradiation image of the rat taken along the path of microbeam propagation. The footprint of the four microbeams is visible on the gafchromic film (rostral-to-caudal penetration pathways of the MTT)

Irradiated cortical field was $4 \mathrm{~mm}$ on the antero-posterior direction (1 $\mathrm{mm}$ anterior to $-3 \mathrm{~mm}$ posterior to the bregma) and, respectively, $3.5 \mathrm{~mm}$ and $7.2 \mathrm{~mm}$ on the lateral direction (starting $1 \mathrm{~mm}$ lateral to midline in the first case and from the midline itself in the second case). X-rays were deposited on the target by vertically moving the kappa goniometer; its speed is inversely proportional to the dose to be delivered. Each irradiation lasted less than $1 \mathrm{sec}$. The vertical irradiation field was determined by the opening-closing of fast shutters, located $7 \mathrm{~m}$ upstream from the rats and synchronized with the movement of the goniometer. All movements were remotely controlled and irradiation values were preset by the operator before the treatment.

The first rat received seven $100 \mu \mathrm{m}$ wide beams with a center-to-center spacing of $400 \mu \mathrm{m}$ and incident (peak) dose of $361 \mathrm{~Gy}$. The dose delivered to the tissue volume in the middle between the beams (valley dose) was limited to $6 \mathrm{~Gy}$. The second rat was exposed to an array of four 600 $\mu \mathrm{m}$ beams with $1200 \mu \mathrm{m}$ spacing. Peak and valley doses of, respectively, $149 \mathrm{~Gy}$ and $6 \mathrm{~Gy}$ were delivered. Valley doses were kept below 6 Gy to avoid the confounding effect on seizure control of higher doses of radiation delivered to the epileptic focus [9]. The sample size for this pilot 
study was limited to two animals due to limited synchrotron facility resource availability and the fact that this initial work was performed primarily to demonstrate feasibility.

The animal immobility during exposure was checked on three control screens located in the control hutch. The incoming spatially non-fractionated dose was measured using an ionization chamber and the mid-valley doses were calculated with Monte Carlo simulations [7]. VEEG recording was continued post-irradiation. Post-hoc seizure quantification was performed by a board certified clinical neurophysiologist. The data was initially processed using Twin (Grass Technologies, West Warwick, RI) software with the following settings:

\section{Seizure detection}

Variation of duration: $36 \%$

Variation of amplitude: $45 \%$

Minimum frequency: $3 \mathrm{~Hz}$

Maximum frequency: $20 \mathrm{~Hz}$

Minimum Bg. amplitude: 2\%

Minimum seizure amplitude: 15\%

Amplitude ratio: 3

Sensitivity: Low

Merge detections: within 1 second

Detect EEG 50\% faster than background

\section{Seizure event}

Generate seizure event every 45 seconds if a seizure is detected or it lasts at least three seconds.

Each seizure event was individually reviewed, and if felt to represent an electrographic ictal event, the total time of the event was counted. If the human reviewer did not agree with a computed detection, it was deleted. Events without clear epileptiform evolution or those lasting less than three seconds were deleted. Video was reviewed for randomly selected seizure events to observe semiologies.

\section{Results}

Electrographic ictal events remained frequent throughout the time period analyzed. Up to 10 hours post-irradiation, rats continued to have electrographic ictal events between $\sim 10-40 \%$ of the recording. Figure 2 illustrates the percentage of time during which ictal electrographic activity was present before and for up to 10 hours post-irradiation. 


\section{Cureus}

$\rightarrow$ Rat 1 (100 $\mu \mathrm{m}$ beams)
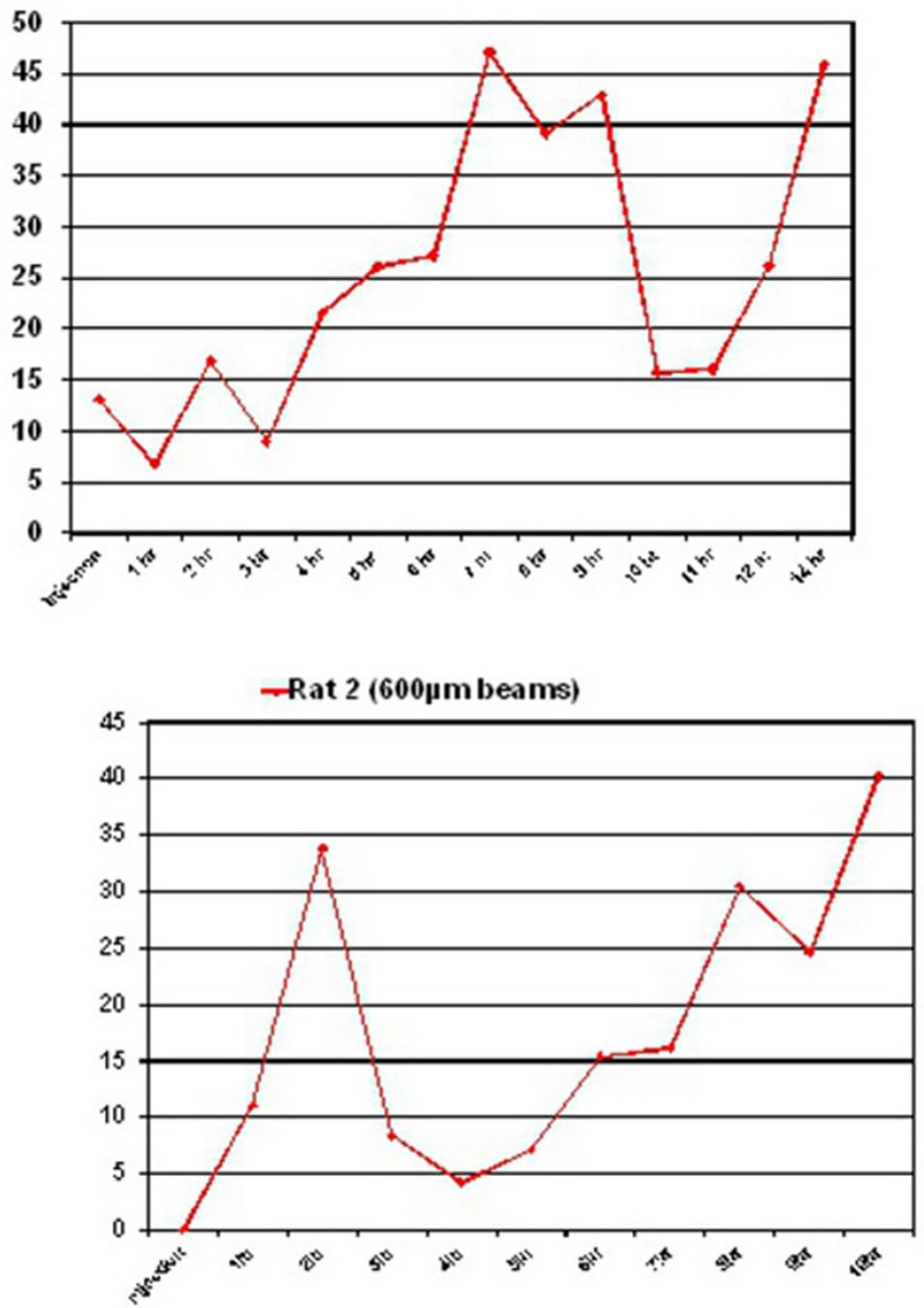

FIGURE 2: Illustration of the percentage of time during which ictal electrographic activity was present before and for up to 10 hours post-irradiation in Rat 1 and Rat 2.

Rat 1 irradiation was performed 215 minutes after kainic acid injection; Rat 2 was irradiated 111 minutes after injection. The results indicate that electrographic ictal events persisted, despite 
abolishment of clinical convulsions following microradiosurgical MST. A sampling of the video was reviewed. Behaviorally, nearly all of these electrographic ictal events were not evident. During the more prolonged seizures, rats exhibited chewing and upper extremity automatisms, cessation of activity, "wet-dog" shakes, or walking around cage. Generalized tonic-clonic seizures were not observed. No sign of contralateral hemiparesis was observed.

\section{Discussion}

Kainic acid has been widely utilized for the induction of seizures in animal models for over 30 years. Mechanisms of its action have been studied in great detail [10]. The key to kainic acid's ability to reliably and potently induce seizures is its effect upon CA3 pyramidal neurons of the hippocampus. At this location, the excitation of kainate-mediated glutamate receptors of mossy fiber synapses takes place. This effect will occur if kainic acid is directly placed into the hippocampus, injected elsewhere within the brain, or administered systemically. Even extremely low concentrations of kainic acid injected into other brain structures will lead to CA3 excitation, likely through activation of afferent pathways. CA3 neurons also function as a pacemaker for synchronized neuronal activity through recurrent collateral synapses. These nascent seizures then spread to CA1 and on to more distal brain regions. The present experiment recorded from bone-screw electrodes and did not include a deep hippocampal electrode. However, semiologically, the seizures we observed suggest hippocampal involvement.

It is likely that very shortly after kainic acid injection there was local excitation of the pyramidal neurons and interneurons located in the vicinity of the sensorimotor cortex. This led to focal cortical excitation and the occurrence of localized ictal activity via synchronized interneuron action potentials. Through neuronal networks, and possibly by direct diffusion of kainic acid, ictal activity eventually began to arise from CA3. These hippocampal-mediated seizures often spread to the point where they were apparent semiologically.

Data reported elsewhere [7] demonstrate that generalized tonic-clonic seizures persisted in similar animals administered kainic acid, but given only sham treatment instead of MTT. The persistence of some electrographic ictal activity with the elimination or near elimination of secondarily generalized seizures may be explained by the action of the MTT. The massive doses of focal irradiation delivered during the MTT may have served to immediately disrupt interneuron networks as well as damage white matter tracts responsible for seizure propagation. The "subclinical" electrographic ictal activity continued as CA3-mediated seizure activity had already begun due to the mechanism described above. These more localized seizures persisted since the hippocampus itself was not within the field of irradiation.

As is evidenced by histology, the MTT successfully transected the cerebral cortex without causing damage to deeper structures. This effect is due to the relatively small half-value layer of low energy microbeams [5]. Therefore, the MTT effectively "parcelized" the seizure focus rather than ablating it. This approach causes no evident neurological damage [7].

Figure 3 is photomicrographs showing immunohistological verification of the irradiation geometry in the cortex. 


\section{Cureus}
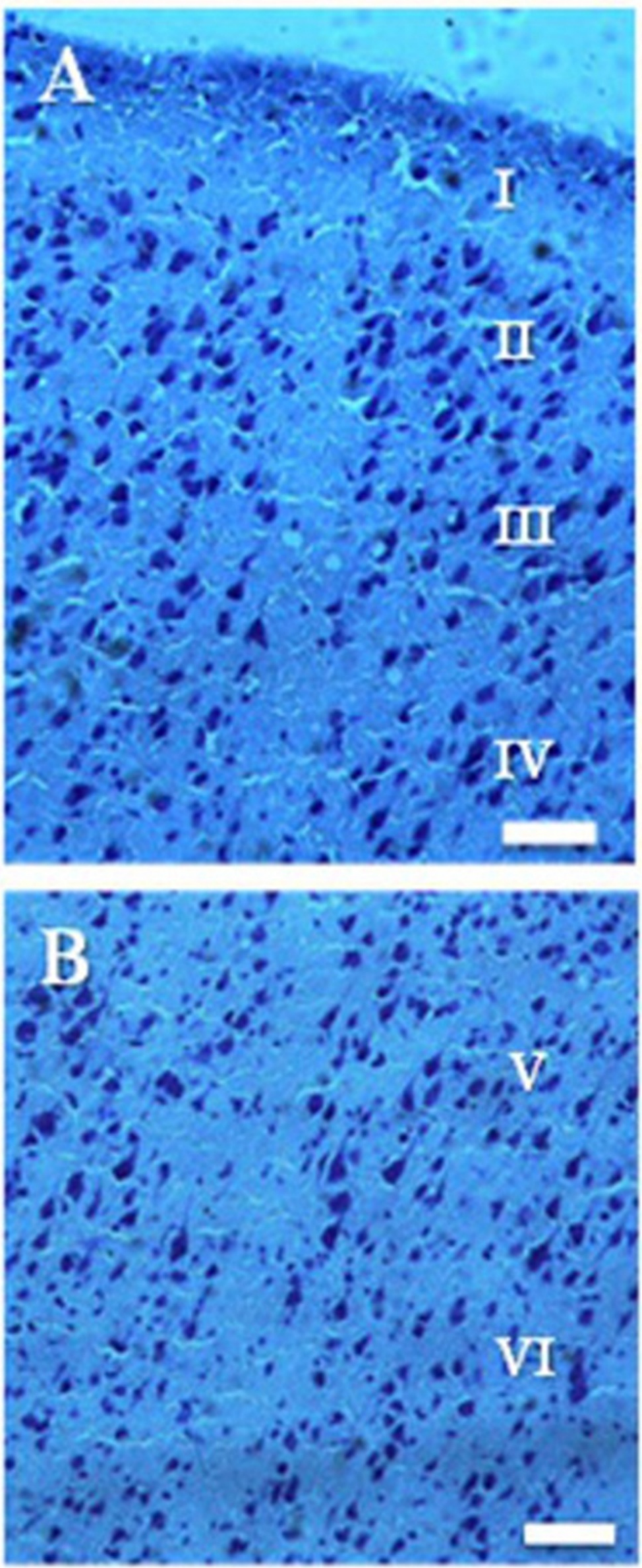

FIGURE 3: Nissl labeling three months after irradiation of the 
Intact pyramidal neurons are clearly visible immediately outside the microbeam path. (Microbeam size: $100 \mu \mathrm{m}$. A,B) Nissl-stained section shows the path of a microbeam crossing levels I to VI; Scale bar: $100 \mu \mathrm{m}$.

\section{Conclusions}

These results demonstrate the feasibility of recording VEEG before and after precise microbeam irradiation from a synchrotron source. VEEG allowed evaluation of electrographic ictal events in addition to clinical seizures. This report details a methodology that may be useful for future animal experiments serving to optimize microbeam doses and configuration in preparation for the treatment of epilepsy in humans. If a technique similar to this is ever to be implemented on a large scale in humans, it will require a less resource-intensive approach than the use of a large synchrotron facility. Further, although limited in scope, the results show some promise that MTT can limit the spread of seizures without causing clinically evident neurological damage.

\section{Additional Information Disclosures}

Human subjects: All authors have confirmed that this study did not involve human participants or tissue. Animal subjects: The study was approved by the European Synchrotron Radiation Facility, Grenoble, France. Issued protocol number protocol number MD-574.

Conflicts of interest: In compliance with the ICMJE uniform disclosure form, all authors declare the following: Payment/services info: All authors have declared that no financial support was received from any organization for the submitted work. Financial relationships: All authors have declared that they have no financial relationships at present or within the previous three years with any organizations that might have an interest in the submitted work. Intellectual property info: Dilmanian FA, Anschel DJ, Gaudette G, Romanelli P, and Hainfeld J. Methods for assisting recovery of damaged brain and spinal cord and treating various diseases using arrays of x-ray microplanar beams. Other relationships: All authors have declared that there are no other relationships or activities that could appear to have influenced the submitted work.

\section{Acknowledgements}

The ESRF is acknowledged for providing their synchrotron radiation facilities, as well as the support and assistance of the ID17 staff and biomedical facility, during all experiments. Sincere gratitude is expressed to H. Requardt, C. Nemoz, Y. Prezado, R. Serduc, D. Dallery, F.

Sebastiano and F. Lena for their individual assistance with experiments. Finally we wish to thank Erminia Fardone of the ESRF for slide preparation

\section{References}

1. Morrell F, Whisler WW, Bleck TP: Multiple subpial transection: A new approach to the surgical treatment of focal epilepsy. J Neurosurg. 1989, 70:231-239.

2. Romanelli P, Striano P, Barbarisi M, Coppola G, Anschel DJ: Non-resective surgery and radiosurgery for treatment of drug-resistant epilepsy. Epilepsy Research. 2012, 99:193-201.

3. Anschel DJ, Bravin A, Romanelli P: Microbeam radiosurgery using synchrotron-generated submillimetric beams: A new tool for the treatment of brain disorders. Neurosurg Rev. 2010, 34:133-142.

4. Bräuer-Krisch E, Serduc R, Siegbahn EA, Le Duc G, Prezado Y, et al.: Effects of pulsed, spatially fractionated, microscopic synchrotron X-ray beams on normal and tumoral brain 


\section{Cureus}

tissue. Mut Res. 2010, 704:160-166.

5. Dilmanian FA, Romanelli P, Zhong Z, Wang R, Wagshul ME, Kalef-Ezra J, Maryanski MJ, Rosen EM, Anschel DJ: Microbeam radiation therapy: Tissue dose penetration and BANG-gel dosimetry of thick-beams' array interlacing. European Journal of Radiology. 2008, 68S:129136.

6. Siegbahn EA, Stepanek J, Bräuer-Krisch E, Bravin A: Determination of dosimetrical quantities used in microbeam radiation therapy (MRT) with Monte Carlo simulations. Medical Physics. 2006, 33:3248-3259.

7. Romanelli P, Fardone E, Battaglia G, Bräuer-Krisch E, Prezado Y, Requardt H, Le Duc G, Nemoz C, Anschel DJ, Spiga J, Bravin A: Synchrotron-generated microbeam sensorimotor cortex transections induce seizure control without disruption of neurological functions. PLOS ONE. 2013, 8:e53549.

8. Spiga J, Siegbahn EA, Bräuer-Krisch E, Randaccio P, Bravin A: The GEANT4 toolkit for microdosimetry calculations: Applications to microbeam radiation therapy (MRT). Medical Physics. 2007, 34:4322-4330.

9. Romanelli P, Anschel DJ: Radiosurgery for epilepsy. Lancet Neurol. 2006, 7:613-620.

10. Ben-Ari Y, Cossart R: Kainate, a double agent that generates seizures: Two decades of progress. TINS. 2000, 23:580-587. 